\section{Don't Be Depth Charged by the Depth Gauge}

Bilal Rafique, Jajini Varghese, Neil Toft

Department of Plastic and Reconstructive Surgery, Royal Free Hospital, London, UK

Correspondence: Bilal Rafique

Department of Plastic and Reconstructive Surgery, Royal Free Hospital, Pond Street, London, NW3 $20 G$ UK

Tel: +44-7599893827, E-mail: bilalrafique90@ hotmail.com

No potential conflict of interest relevant to this article was reported.

Received: 12 Jan 2017 • Revised: 5 Feb 2017 • Accepted: 6 Feb 2017 pISSN: 2234-6163・ elSSN: 2234-6171

https://doi.org/10.5999/aps.2017.44.4.356 • Arch Plast Surg 2017;44:356-357

(a)

Copyright (C) 2017 The Korean Society of Plastic and Reconstructive Surgeons

This is an Open Access article distributed under the terms of the Creative Commons Attribution Non-Commercial License (http://creativecommons.org/licenses/by-nc/4.0/) which permits unrestricted non-commercial use, distribution, and reproduction in any medium, provided the riginal work is properly cited.

\section{Dear Sir/Madam,}

The depth gauge is an essential device used while performing open plate or interosseous screw fixation of hand fractures allowing for anatomically accurate and safe insertion of screws. An efficient pass through the drilled path provides the best chance at accurate estimation of correct screw length. The most common error is inaccurate measurement of the length of the drilled hole, which subsequently

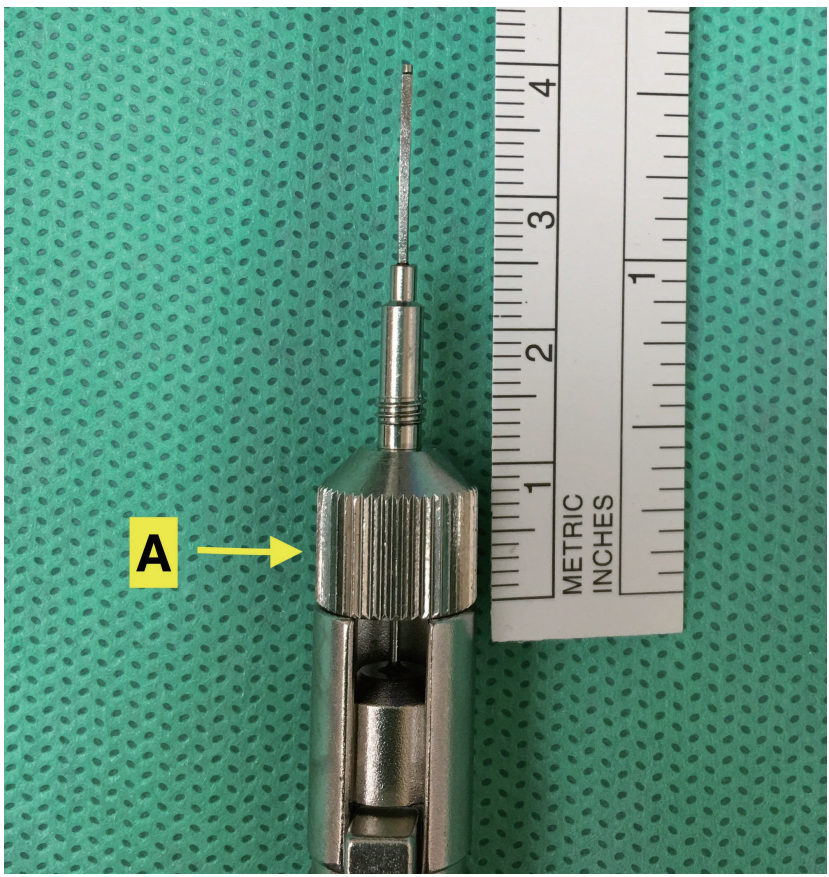

Fig. 1.

Correct assembly of depth gauge. Label A, metal collar. leads to inaccurate screw choice. Too long a screw with over projection may cause attrition and rupture of tendons; too short may not "bite" and engage the distal cortex resulting in instability. We wish to highlight a potential source of error with the use of this device.

Occasionally the lip of the depth gauge may slip back through the volar cortical edge or in some cases catch the inner dorsal cortex. In these circumstances the surgeon is usually aware of its incorrect placement based on tactile feedback. However in cases where there is no doubt that sufficient traction on the volar cortex has been achieved a length discrepancy may still remain. Fig. 1 demonstrates a depth gauge with measurement of the slim steel bar from the proximal point of the sliding metal collar (labelled A), this correlates with measurement along a graduated bar indicating length of screw required. A slight loosening of the screw mechanism (labelled B, Fig. 2 ) on which the metal collar is mounted proportionately affects the length reading obtained as shown in Fig. 2. This may be in the order of only one millimetre or more but has consequence on choice of screw. It is particularly deceiving when good distal grip is obtained while carrying out measurement. This leads the way for insertion of an inaccurately long screw, a fact which would only come to light once lateral or oblique radiographic imaging has been obtained as in Fig. 3. As a result the screw is required to be replaced by one of appropriate length, compromising the threaded trail along the canal and possibly leading to suboptimal screw grip and the need for a larger threaded "rescue" screw.

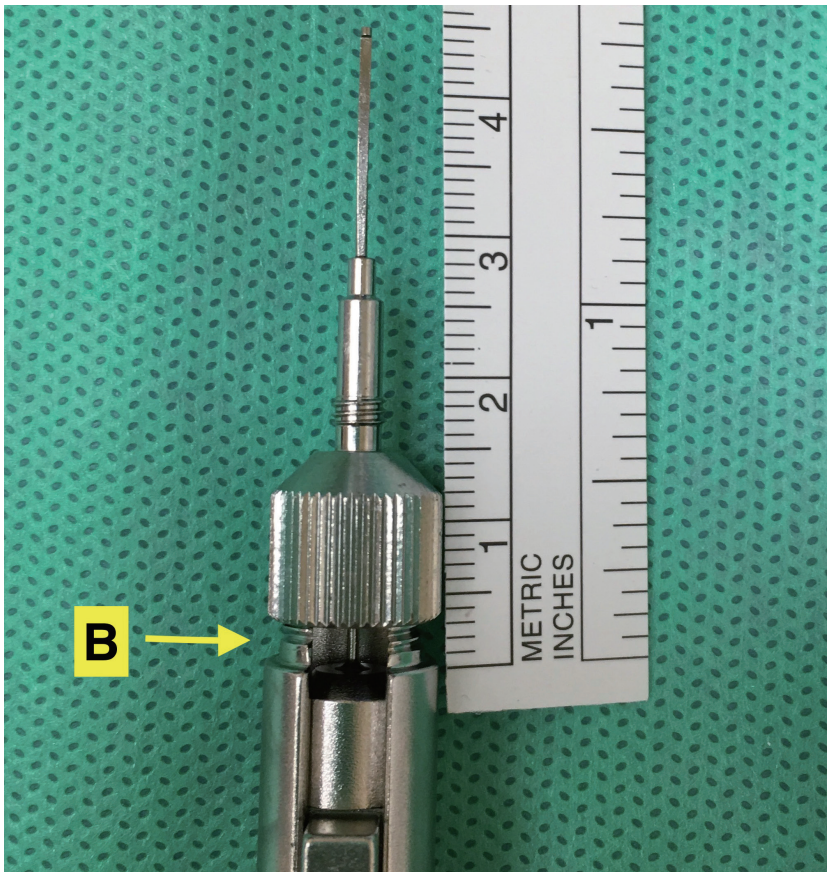

Fig. 2.

Incorrect assembly of depth gauge metal collar not screwed tightly following cleaning in sterile services. Label $B$, a loosening of the mechanism. 


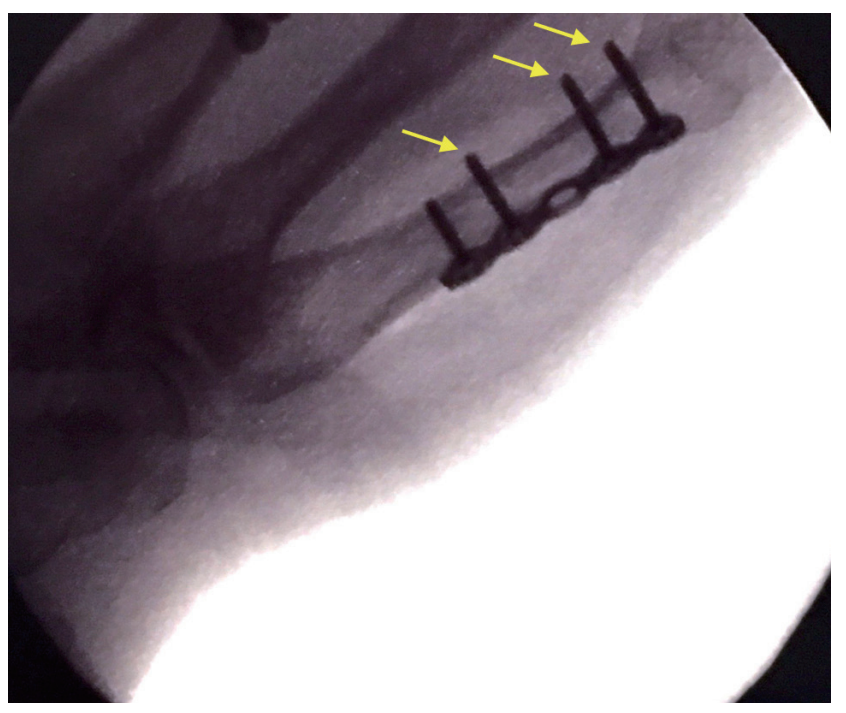

In summary, accurate drill hole measurement using a depth gauge is crucial in order to choose the anatomically correct screw length. The metal collar of this device, if not firmly in position, may lead to an apparently long measurement. Knowledge of this key but easily overlooked point will help in obtaining true length and subsequent correct screw sizing and passage in a single stage helping to optimize fracture fixation.

Fig. 3.

Radiograph demonstrating over projected screws (yellow arrows) as a result of displaced metal collar. 